\title{
BMJ Open Evaluating the importance of policy amenable factors in explaining influenza vaccination: a cross-sectional multinational study
}

\author{
Ana Wheelock, ${ }^{1}$ Marisa Miraldo, ${ }^{2}$ Angus Thomson, ${ }^{3}$ Charles Vincent, ${ }^{4}$ \\ Nick Sevdalis ${ }^{5}$
}

To cite: Wheelock A, Miraldo M, Thomson A, et al. Evaluating the importance of policy amenable factors in explaining influenza vaccination: a cross-sectional multinational study. BMJ Open 2017;7:e014668. doi:10.1136/ bmjopen-2016-014668

- Prepublication history and additional material for this paper are available online. To view these files please visit the journal online (http://dx.doi. org/10.1136/bmjopen-2016014668).

Received 11 October 2016

Revised 4 May 2017

Accepted 1 June 2017

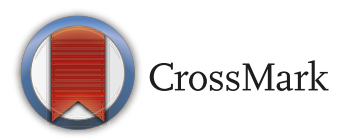

${ }^{1}$ National Institute for Health Research Imperial Patient Safety Translational Research Centre (PSTRC), Imperial College London, London, UK

${ }^{2}$ Imperial College Business School, London, UK

${ }^{3}$ Sanofi Pasteur, Lyon, France

${ }^{4}$ Department of Experimental

Psychology, University of Oxford Oxford, UK

${ }^{5}$ Centre for Implementation

Science, Health Service and Population Research Department, King's College London, London, UK

Correspondence to

Dr Ana Wheelock; a.wheelock@ imperial.ac.uk

\section{ABSTRACT}

Objectives Despite continuous efforts to improve influenza vaccination coverage, uptake among highrisk groups remains suboptimal. We aimed to identify policy amenable factors associated with vaccination and to measure their importance in order to assist in the monitoring of vaccination sentiment and the design of communication strategies and interventions to improve vaccination rates.

Setting The USA, the UK and France.

Participants A total of 2412 participants were surveyed across the three countries.

Outcome measures Self-reported influenza vaccination. Methods Between March and April 2014, a stratified random sampling strategy was employed with the aim of obtaining nationally representative samples in the USA, the UK and France through online databases and random-digit dialling. Participants were asked about vaccination practices, perceptions and feelings. Multivariable logistic regression was used to identify factors associated with past influenza vaccination. Results The models were able to explain $64 \%-80 \%$ of the variance in vaccination behaviour. Overall, sociopsychological variables, which are inherently amenable to policy, were better at explaining past vaccination behaviour than demographic, socioeconomic and health variables. Explanatory variables included social influence (physician), influenza and vaccine risk perceptions and traumatic childhood experiences. Conclusions Our results indicate that evidence-based sociopsychological items should be considered for inclusion into national immunisation surveys to gauge the public's views, identify emerging concerns and thus proactively and opportunely address potential barriers and harness vaccination drivers.

\section{BACKGROUND}

Upper respiratory tract infections are a leading cause of mortality and morbidity in high-income countries, mostly among adults. ${ }^{1}$ Influenza is a major contributor to this burden of disease; estimates show that up to 49000 people die every year in the $\mathrm{USA}^{2}$ and 40000 in the European Union $^{3}$ from influenza-related illness.

\section{Strengths and limitations of this study}

- We generated regression models comprised of a broad set of variables, most of which have been linked to vaccination behaviour.

- We also aimed to use representative samples of the population of interest in three different developed countries (the USA, the UK and France).

- The employed survey measures concerned the individual and conditioned perceptions on their vaccination status.

- Our research may have suffered from respondentrelated biases. For example, people for whom vaccination issues are particularly salient may have been more prone to participate.

In most developed economies, an annual influenza vaccine is recommended and offered free of charge to those at higher risk of death from influenza complications, including pregnant women, individuals with eligible chronic illnesses and people aged 65 years and older. The vaccine is also available at a cost-usually in pharmacies or private healthcare facilities-to those who do not belong to a risk group, but wish to protect themselves. In the USA, for example, where the vaccine is recommended to all adults, approximately one-third of healthy adults under 65 years old vaccinate against influenza every year. ${ }^{4}$

Despite continuous efforts to improve influenza vaccination coverage, uptake among high-risk groups remains low. In 2013/2014, for example, $65 \%$ of older adults $(\geq 65 \mathrm{~s})$ and $46 \%$ of younger adults with eligible health conditions were vaccinated against influenza in the USA. ${ }^{4}$ In the same season, vaccination rates in the UK, one of the highest in Europe, were $73 \%$ in $\geq 65 \mathrm{~s}$ and $53 \%$ in eligible under $65 \mathrm{~s}$, both below the minimum $75 \%$ coverage recommended by the WHO. ${ }^{5}{ }^{6}$ Worryingly, 
a $151 \%$ rise in excess winter deaths in England and Wales in 2014/2015, partly attributed to the circulation of a mutated A(H3N2) influenza strain which made the vaccine significantly less effective, ${ }^{7}$ alongside unseasonable warm weather in 2015/2016, resulted in the lowest vaccination uptake in more than a decade. ${ }^{8}$ Vaccination decisions are shaped by a myriad factors, including demographic, socioeconomic and sociopsychological factors. ${ }^{9-12}$ The latter are of particular interest, given that they are inherently amenable to policy and interventions to change behaviour. Yet, few countries routinely collect data on people's beliefs and perceptions towards vaccination, and those that do often use one open question (eg, 'Why didn't you get a flu shot last winter?'). ${ }^{13}$ Although cheaper and easier to administer, this form of enquiry does not take into account people's tendency to fall back on readily available information (eg, the first thought that comes to mind) or report postdecisional rationalisations of their behaviours (eg, 'I did not vaccinate, hence it must not be necessary') rather than actual drivers. ${ }^{14} 15$ Moreover, these data do not allow comparative analyses between vaccinated and unvaccinated people.

Multilateral efforts to measure and improve confidence in vaccines are gathering pace, ${ }^{16}{ }^{17}$ yet they are built upon a body of evidence which, although extensive and insightful, has a number of gaps. One key limitation is that many studies evaluating the link between sociopsychological factors and influenza vaccination do not use multivariable analysis, thus the importance of a given variable in relation to others often remains unknown. Studies that do employ multivariable analysis seldom perform (or report) robustness checks and usually comprise a limited number of variables, which can result in omitted-variable bias, where the model compensates for the missing variables by over or underestimating the effect of the included variables. ${ }^{9} 1819$ For example, omitted-variable bias could explain why the model developed by Weinstein et al-comprised of seven variables-showed that anticipated regret of not vaccinating was more important than other established influenza perceptions or why they did not find an association between vaccine effectiveness and vaccination uptake in this US sample. ${ }^{18}$ Moreover, these studies frequently include proxies of vaccination uptake, such as historical vaccination (ie, vaccination in previous seasons not including the most recent) in the case of retrospective studies or intention to vaccinate in the case of prospective studies, as independent variables, ${ }^{9} 1920$ thereby artificially boosting the explanatory ability of the model-because most people who vaccinate against influenza do so periodically-without necessarily explaining vaccination behaviour ( $\mathrm{eg}$, people vaccinate because they feel vulnerable and/or receive a reminder from their general practitioner (GP) every winter). As Brewer and colleagues note, other important methodological shortcomings are the prevalent use of weak survey measures (eg, generic risk perceptions rather than own perceived risk) and small convenience samples, which may affect the validity and generalisability of findings. ${ }^{11} \mathrm{~A}$ related drawback is that most of the evidence in this area is produced in the USA, thus important contextual issues remain unexplored. Furthermore, vaccination coverage and factors underpinning uptake among healthy adults are often unknown.

We sought to address these limitations by generating regression models comprised of a broad set of variables, most of which have been linked to vaccination behaviour, by employing measures that gauge individuals' own perceived risk (eg, 'The flu could make me severely ill') and condition their perceptions upon having or not having received the vaccine (eg, 'With no flu vaccine, I would feel very vulnerable to the flu'), ${ }^{11}$ and aiming to use representative samples of the population of interest in three different developed countries: the USA, the UK and France. In order to assist in the monitoring of vaccination sentiment and the prioritisation and design of communication strategies and interventions to increase influenza vaccination across different contexts, this study aimed to answer three research questions: (1) What are the variables that consistently explain recent influenza vaccination uptake? (2) What is the importance of policy amenable factors in relation to demographic, socioeconomic and health characteristics in explaining past vaccination behaviour? (3) Are the factors associated with influenza vaccination comparable across countries?

\section{METHODS}

\section{Study sample}

Using stratified random sampling, we aimed to survey nationally representative adult samples from the USA, the UK and France, about vaccination between March and April 2014. Interlocking quotas based on gender, age and income were set. In addition, to ensure national representativeness, regional, settlement type (rural/urban) and ethnicity non-interlocking quotas were put in place.

Since some of the included variables had not been previously tested and others were not consistently correlated with vaccination in previous studies, we assumed that the correlation coefficient between dependent and independent variables was 0.1 (a small effect size), the minimum sample was calculated to be 782 subjects per country $(\alpha=0.05 ; 1-\beta=80 \%)$ with PASS V.11.

The American Institutes for Research (USA) and the Imperial College Research Ethics Committee (UK) granted research ethics approval. The French Commission nationale de l'informatique et des libertés and Comités de protection des personnes granted waivers to approval. Participants were informed about the nature of the study and provided consent.

\section{Procedure}

A market research company (Double Helix) was responsible for piloting, programming the online survey and conducting the telephone interviews. Ten pilot interviews (seven face-to-face and three telephone interviews) were conducted with purposively selected participants in 
the UK to test the survey's face and content validity, and ease of completion. Additionally, 10 pilot interviews were conducted over the phone in the USA and 10 in France with the aid of a screen sharing platform. Interviews were conducted by a trained researcher while the rest of the team observed via live broadcast. The pilot showed the survey was easy to complete and understand, and lasted approximately $20 \mathrm{~min}$. The refinements to the study materials were related to wording and format. Self-completion online surveys were then sent to a non-probability online panel and random-digit dialling was employed to recruit a proportion of the $65+$ age category and those belonging to $\mathrm{D} / \mathrm{E}$ socioeconomic groups, due to their limited access to or lack of familiarity with internet-based applications ${ }^{21}$ (see online supplementary box S1 for more details about non-probability online panels).

As a quality control measure, participants classified as 'speeders' (completed the survey in half of the average length-16 min) and 'flat-liners' (gave homogenous responses and completed the survey in less than half of the optimum survey length-20 min) were removed and replaced. ${ }^{22}$

\section{Instrument}

The measures reported here are a subset of a larger vaccination survey (available from the authors upon request). Our analyses included 32-34 items (see online supplementary table S1). We selected sociopsychological items that had been linked to influenza vaccination based on existing evidence. These comprised adapted constructs from the Health Belief Model $^{23}$ and Protection Motivation Theor ${ }^{24}$-notably, influenza and vaccine risk perceptions, vaccine effectiveness and self-efficacy, ${ }^{9-12} 25$ perceived knowledge of the vaccine ${ }^{10}$ and items assessing trust in key vaccination stakeholders ${ }^{26}$ Additional policy amenable factors which had infrequently been used in the context of vaccination, but were considered potential explanatory variables, were also tested. These were worry of infecting other people (if unvaccinated) ${ }^{27}$-a measure aimed at evaluating the extent to which people vaccinate to protect others, perceived control over influenza, ${ }^{28} 29$ regret of contracting influenza, ${ }^{30}$ childhood traumatic health experiences ${ }^{31}$ - to evaluate their influence on adult vaccination behaviour, and health decision-making preferences $^{32}{ }^{33}$ - to further explore the effect of the doctor-patient relationship on vaccination acceptance. Participants' socioeconomic, demographic and health characteristics previously associated with influenza vaccination were prioritised. ${ }^{934}$

We used 11-point Likert scales (0-10) for the majority of sociopsychological items, as these are recognised for their reliability and ease of completion, ${ }^{35}$ and multiple-choice items and alternate-choice items when appropriate. Except for trust, health decision-making preferences and childhood traumatic health experiences items, sociopsychological measures were disease or vaccine specific to avoid misinterpretation. As illustrated in the introduction, our questions also aimed to capture the respondent's perception of their own personal risk rather than their views on risk of illness in the wider population. Thus, we asked how likely it is that they might become ill rather than how likely people generally are to get influenza. We also wished to specifically focus their attention on the risk of influenza in the presence or absence of vaccination, as people may feel more or less protected depending on their vaccination status. The questions were therefore in the form of 'Without a vaccine, it is likely I will get the flu' rather than simply assessing their views on the likelihood of getting influenza. Finally, when thematic hierarchy (eg, from general to specific) was not important, items were rotated to minimise response bias.

\section{Data analysis}

We used the following formula to calculate response rates: number of surveys completed divided by sent emails or interviews attempted minus ineligible individuals, multiplied by 100 . Descriptive statistics, Pearson's $\chi^{2}$ test and t-tests were computed to explore the relationships between the assessed variables and self-reported past vaccination behaviour. Point-biserial correlations were calculated and $\chi^{2}$ statistics were converted into correlation coefficients to explore whether the relationship between the dependent and independent variables matched or exceeded a coefficient of 0.1 -the assumption employed to calculate the sample size. Cronbach's alpha was used to explore the reliability of the proposed measures across countries. The outcome measure was receiving an influenza vaccine in the last 6 months (2013/2014 influenza season).

Given that the dependent variable was binary, logistic regression analysis was conducted to identify the variables associated with past influenza vaccination. Four continuous variables with 'I don't know/not applicable' responses were dichotomised as follows: values expressing agreement with a given statement (6-10) were coded as $1=$ 'yes' and the rest $(0-5$ and 'I don't know/not applicable') were coded as $0=$ 'other than yes' (see online supplementary tables S2-S4).

Although a software-based stepwise approach is widely used in logistic regression, in recent years the purposeful selection of variables has been favoured over deterministic model-building methods. This is because the latter tend to rely on automatic selection of variables based only on mathematical criteria, which can lead to overfitting or underfitting models. Therefore, we used a manual stepwise, hierarchical approach, where blocks of variables were entered in a sequence based on previous evidence and our aim of assessing the importance of policy amenable factors in explaining influenza vaccination (see online supplementary box S2 for a full description of the approach) ${ }^{36}$

Two goodness-of-fit tests- $\chi^{2}$ and Nagelkerke $\mathrm{R}^{2}$-were used to assess the overall model (M1) and each of the seven models (blocks) generated using the hierarchical approach. Employing a classification cut-off point of 0.5, a final model with a Nagelkerke $\mathrm{R}^{2}$ value close to 1 , which indicates optimal model fit, was sought. 
Thorough checks to ensure the robustness of the models were conducted, including variance inflation factor (VIF) to assess collinearity, standardised residuals to detect and evaluate outliers and Cook's distance to identify influential cases. Separate analyses entering the blocks of variables in reverse order were also performed (ie, from block 7 to block 1) to evaluate whether the order in which variables were entered significantly modified our results. Data were analysed using IBM SPSS Statistics V.22.

\section{RESULTS}

\section{Participants}

The online survey was completed by 814 participants in the USA, 791 in the UK and 787 in France. Online response rates were low $(\mathrm{USA}=1 \%$; UK $=1.7 \%$; France $=2.4 \%$ ), although consistent with research on non-probability online panels showing that, in recent years, response rates have fallen to a point where in many cases they are $10 \%$ or less. ${ }^{37}$ Eighty participants were interviewed via the telephone in the USA, 100 in the UK and 100 in France. Telephone response rates were 6\%-9\%. Telephone interviews targeted older people and those belonging to low socioeconomic strata, two populations with particularly low response rates. ${ }^{38}$ Recruitment flow diagrams for the online and telephone samples are presented in online supplementary figures S1A-S3A and S1B-S3B, respectively.

Except for education in the USA-the sample was more educated than the general population, there were no significant differences between the characteristics of the final samples (USA=801; $\mathrm{UK}=806$; France $=805$; total sample $n=2412$ ) and those of the general population, when available (table 1 ). To facilitate survey completion and improve data accuracy, household income data for this study were collected using a limited number of bands relevant to each country. Therefore, it cannot be directly compared against census data, which collects more granular income information per household. However, we have used as a reference the band that is most approximate to the census median household income (table 1). In the USA and the UK, roughly half of the sample was below the reference band and the other half was above; whereas in France, the number of participants who reported a household income below the median was substantially higher than those over the median (table 1).

Healthcare professionals were excluded from the final samples as their decision-making processes are influenced by those they care for or regulated by healthcare authorities, thus some of their motivations and concerns may differ from those of the general population. ${ }^{39}$ Subgroup analyses confirmed these differences (available upon request).

\section{Differences between vaccinated and non-vaccinated participants}

Overall, the responses of vaccinated and unvaccinated participants were significantly different $(\mathrm{p}<0.05-0.001)$ and comparable across countries (see online supplementary tables S2-S4). Those who had received an influenza vaccine were older, reported having an eligible health condition, had a private or public health insurance, lived with a partner (USA/France), were wealthier (USA/ France) and more educated (USA). They were also less constrained by practical barriers and more likely to report that their physician and relatives thought they should vaccinate than those who had not received a vaccine. Vaccinated participants were more concerned about the risks of influenza, less worried about the risks of the vaccine and more trusting of vaccine manufacturers and providers than unvaccinated participants. Vaccinees reported possessing a better understanding of the influenza vaccine and were more prone to let physicians make decisions about their health (USA/UK) than those who had not vaccinated. Lastly, vaccinated participants were less likely to have had a bad vaccine or injection-related experience (UK) and more likely to have had a scary health-related experience in childhood than unvaccinated participants.

\section{Factors associated with past influenza vaccination in regression analyses}

When all variables were assessed concurrently, the models that best fitted the data (M6-M8) explained 73\% of the variance in past vaccination behaviour in the USA, $80 \%$ in the UK and $64 \%$ in France (Nagelkerke $\mathrm{R}^{2}=0.644-0.795$ ) (tables 2-4). The first models (M1) included all the variables, thus were less parsimonious than M6-M8, yet they explained a similar share of the variance $(66 \%-80 \%)$. When using the hierarchical approach, the first-step models (M2), which included demographic, socioeconomic and health variables, fitted the data poorly to moderately and accounted for $22 \%$ of the variance in past vaccination behaviour in the USA, 38\% in the UK and 19\% in France. Practical barriers only explained 3\% of the variance in the USA (M3) and were not significant in the UK and France. Social influence explained 14\% of the variance in the USA (M4), $21 \%$ in UK and $25 \%$ in France (M3). Influenza perceptions accounted for $30 \%$ of past vaccination behaviour in the USA (M5), $17 \%$ in the UK and $18 \%$ in France (M4), whereas influenza vaccine perceptions only explained $1 \%$ of this behaviour in the USA (M6), 2\% in the UK and 1\% in France (M5). Finally, trust items explained less than $1 \%$ of the variance in the USA, while decision-making preferences and childhood experiences explained $2 \%$ of the variance in the UK and $1 \%$ in France.

When blocks were entered in reverse order, demographic, socioeconomic and health variables contributed little to the variance in past vaccination behaviour-3\% (USA), 1\% (UK) and 0\% (France). This is not surprising, since people's characteristics have an effect on their perceptions, thus they explain some of the same variance. This result further proves that poorly specified models-which are not evidence based-lead to biased estimates (the detailed results 


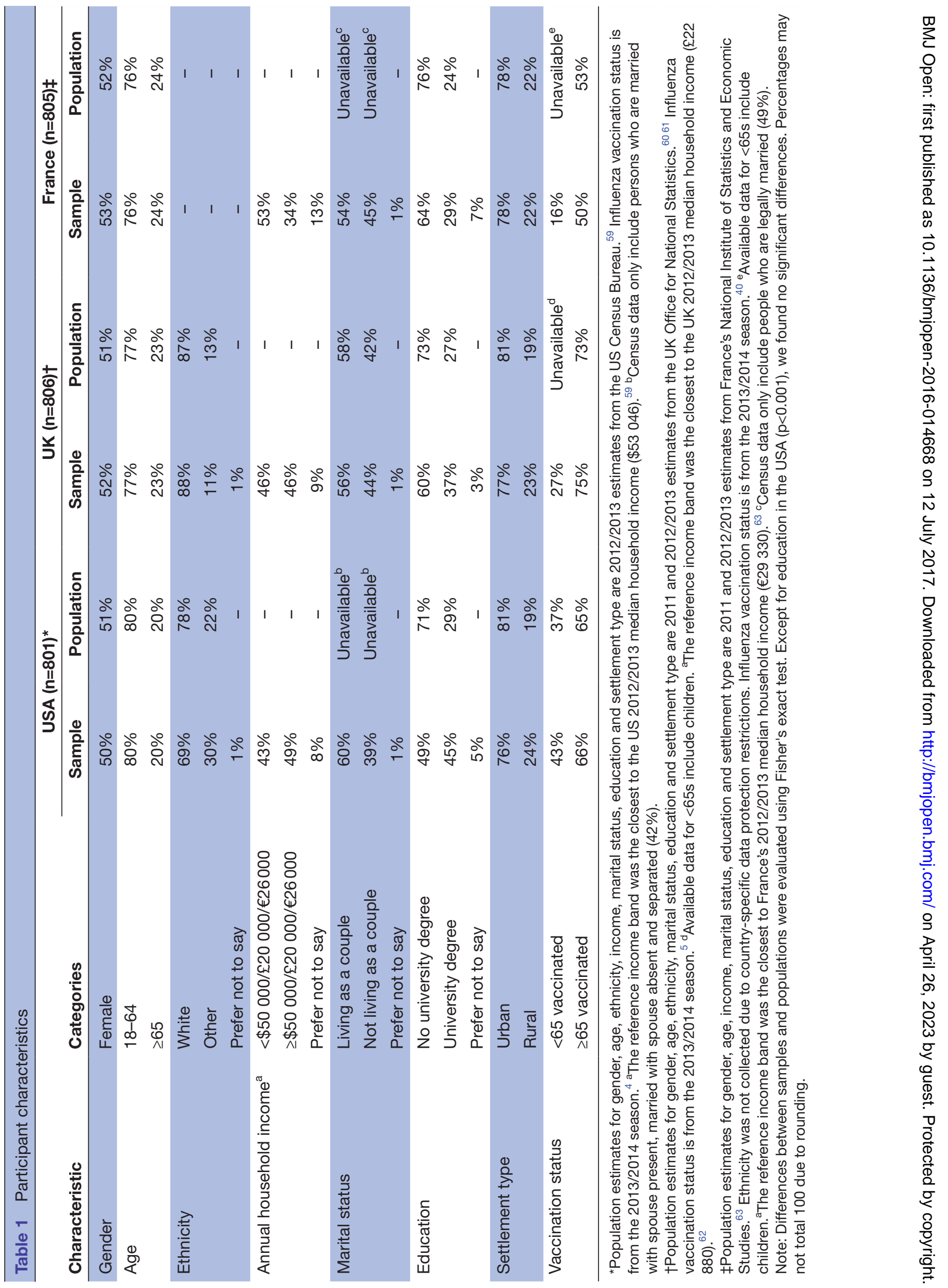




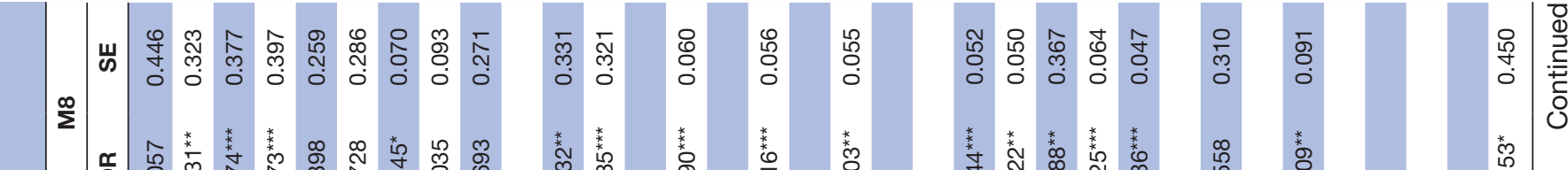

๘

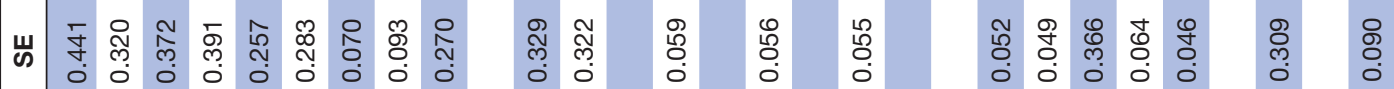

$\hat{\Sigma}$

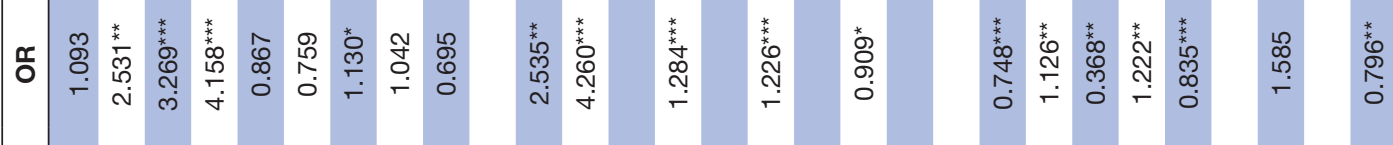

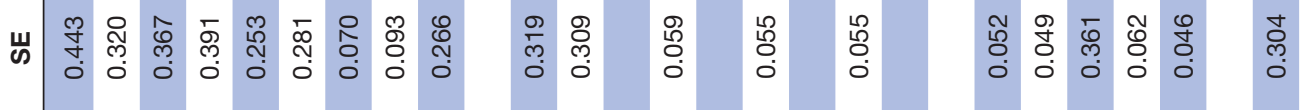

$\stackrel{\bullet}{\Sigma}$

o

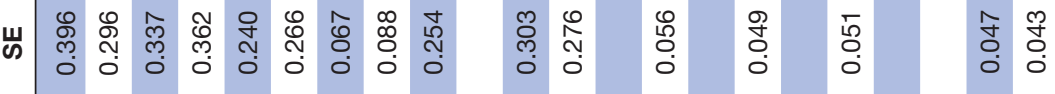

刋

q

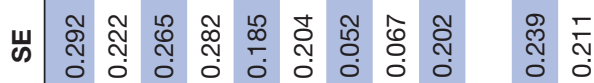

$\stackrel{\text { 范 }}{\Sigma}$

$\dot{\Sigma}$

ल)

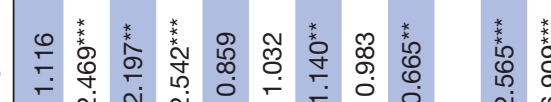

$\frac{n}{n}$

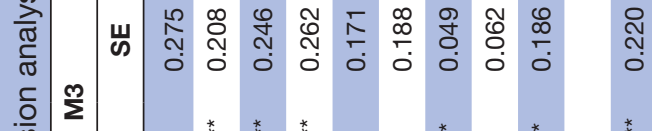

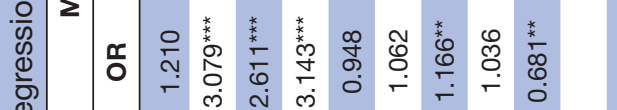

事

든

. $\frac{\sqrt{0}}{\mathrm{C}} \mathrm{N}$

س

药

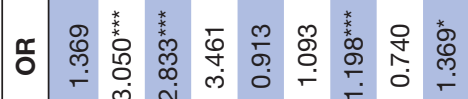

N

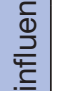

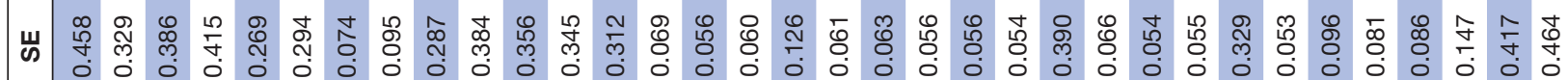

雚

受

。

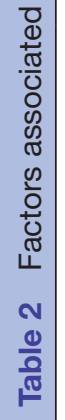

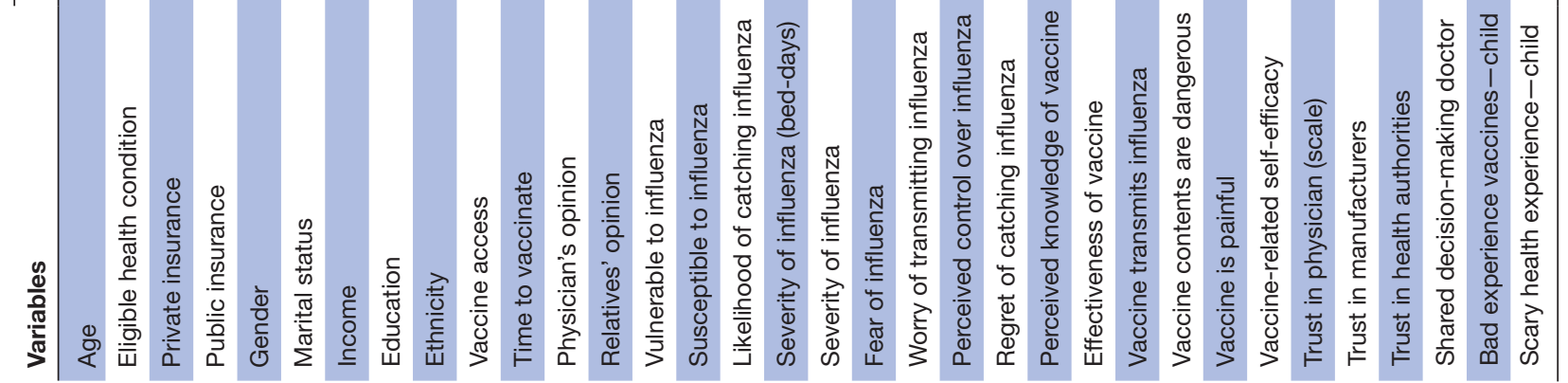




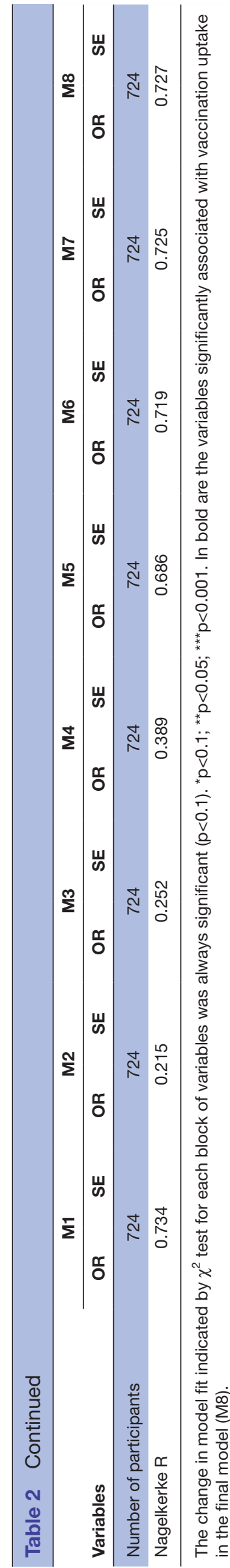

of these analyses are available from the corresponding author upon request).

Robustness checks showed that the variables which were significant in M1 remained significant across most 11-21 specifications, with some exceptions. In the USA, 'vaccine is painful' became non-significant when non-significant influenza perceptions were removed. This suggests that the latter had a suppressor effect on the former, that is, their inclusion strengthened the effect of the variable in question. ${ }^{40}$ In the UK, gender became significant when non-significant vaccine perceptions were removed, and 'vaccine transmits influenza' became significant when 'vaccine contents are dangerous' was removed. In both cases, this indicates that the removed variables were confounders of those that became significant. ${ }^{41}$ In France, 'trust in manufacturers' was a confounder of education-the latter became significant in the absence of the former, and 'trust in physician' was a suppressor of 'vaccine access' - the latter became non-significant when the former was excluded. ${ }^{4142}$ Consequently, all the variables that were significant in M1, one non-significant variable that became significant while performing robustness checks ('vaccine transmits influenza' in the UK) and all the controls were included in the hierarchical models. The magnitude and significance of the relationship between independent and dependent variables varied little between the first models (M1), where all the variables were entered at the same time, and the last models (M6-M8), where a reduced number of variables were entered in blocks, which is a further indication of the robustness of our findings. Detailed robustness checks are not presented here for brevity, but are available from the corresponding author upon request.

All the correlation coefficients between the dependent and the independent variables were higher than 0.1 , except for two variables which were tested for the first time in this study: 'Bad experience vaccines - child' $(\mathrm{r}=$ $-0.082, p<0.05$ in the UK; $r=0.040, p>0.05$ in the USA; and $\mathrm{r}=-0.064, \mathrm{p}>0.05$ in France) and 'Scary health experience - child' ( $r=0.090, p<0.05$ in the USA) (detailed results are available from the corresponding author upon request).

Cronbach's alpha coefficients ranged from acceptable $(\alpha=0.65)$ to excellent $(\alpha=0.87)$ and they were comparable across countries for each evaluated scale, except for 'trust in vaccination stakeholders,' which was considerably less reliable in France (see online supplementary table S5). Overall, these results indicate that the scales worked in a similar manner across the three countries. Further psychometric analyses and scale refinement will be performed and reported in a separate article.

Collinearity diagnostics showed that all variables had VIF values below 5 , indicating there is no cause for concern. ${ }^{43}$ Standardised residuals were also examined to identify outliers. Less than $5 \%$ of the cases had standardised residuals above 2 and no more than $1 \%$ had absolute values higher than 3, thus there was no need to eliminate or transform cases. ${ }^{44}$ Cook's distance statistics were evaluated to identify cases exerting excessive influence on the 


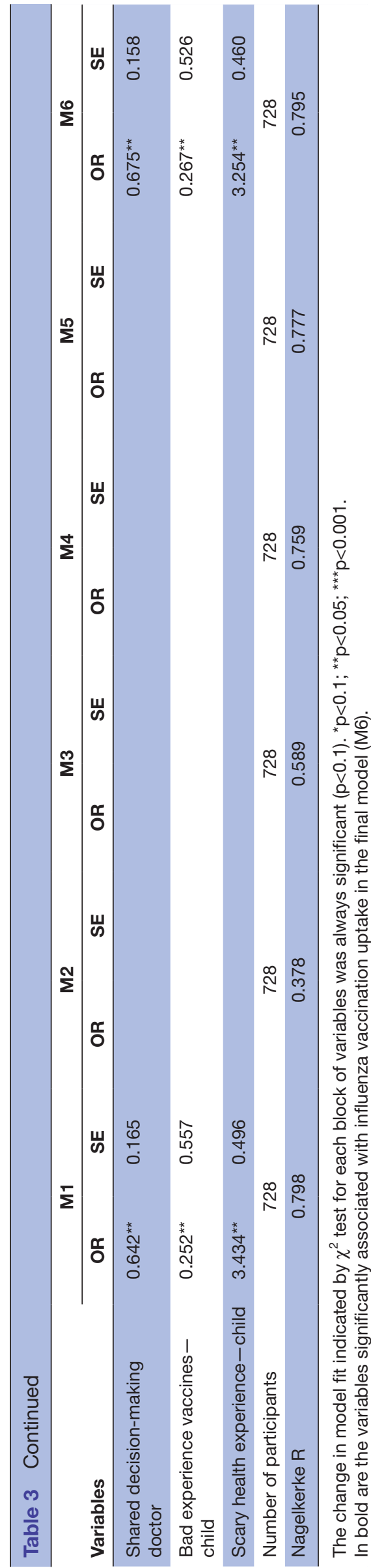

model. No values were higher than 1 , which shows that no case had to be excluded on that basis. ${ }^{45}$

\section{DISCUSSION}

This study aimed to identify policy amenable factors associated with recent influenza vaccination uptake among adults in three high-income countries and to quantify their impact. Our results support previous findings and add new insights.

The final models robustly explained $64 \%-80 \%$ of the variance in past vaccination behaviour and although some predictors were country specific, we found important commonalities (table 5). To the best of our knowledge, ours is the first study to demonstrate that sociopsychological variables consistently explain most of the variance in past influenza vaccination behaviour, over and above demographic, socioeconomic and health variables $(49 \%$ vs $22 \%$ in the USA, $42 \%$ vs $38 \%$ in the UK and $45 \%$ vs $19 \%$ in France). Our findings also show that the most important policy amenable factors were social influence, particularly physicians' (USA $=14 \%, \mathrm{UK}=21 \%$ and France $=25 \%$ of the variance) and perceptions about influenza (USA $=30 \%, \mathrm{UK}=17 \%$ and France $=18 \%$ of the variance), communication efforts should, therefore, focus on these factors. Surprisingly, perceptions about the influenza vaccine explained a very small proportion of vaccination behaviour across the three countries. Additionally, our results show that a sizeable proportion of adults under the age of 65 years, both with and without eligible chronic conditions, is vaccinating against influenza in the USA (over a third) and the UK (under a third), while only $16 \%$ do so in France.

Specifically, and in line with previous evidence, we found that age, health status, health insurance, income, gender, marital status and education were associated with past vaccination. ${ }^{934}$ Differences between countries are likely influenced by their healthcare systems and immunisation policies.

For example, having an eligible health condition was more important than age on its own in the USA and the UK, whereas the opposite occurred in France. One plausible reason is that a controversy about the effectiveness and safety of the $\mathrm{A}(\mathrm{H} 1 \mathrm{~N} 1)$ pdm09 vaccine in 2009/2010, which has had a lasting negative impact on seasonal influenza vaccination rates in France, may have dissuaded some populations-such as younger people with and without eligible health conditions who may feel less vulnerable-more than others. ${ }^{34} 46$ This controversy may also be underpinning the differences in model variance and reliability of the trust scale found between France and the other two countries, both of which had not experienced important influenza vaccination scares in recent years, and hence, had maintained fairly constant vaccination rates for more than a decade at the time of data collection. ${ }^{4512}$ Private and public health insurance, and income were associated with past vaccination in the USA, a country with a largely privatised healthcare system. 
Although the UK and France have healthcare systems which are free at the point of delivery or affordable for most, the influenza vaccine is only free of charge for highrisk groups, which may explain the association between health insurance and past vaccination in both countries-although weak in France. Marital status was also correlated with past vaccination in the UK and France. Higher vaccination rates among participants living with a partner may be explained by people's tendency to protect their significant other or encouragement from partners to get vaccinated, yet more evidence is needed to substantiate this assertion. Finally, being male and more educated were positively associated with past vaccination in the UK and France, respectively. Yet, both characteristics were not robustly correlated with past vaccination across all specifications, and the association between gender and vaccination in the UK is weak, thus these findings should be interpreted with caution. Future research testing our findings across adequately powered samples of high-risk people will certainly improve our understanding of the relative importance of demographic, socioeconomic and health factors in vaccination decisions among eligible individuals. We hypothesise that sociopsychological factors are likely to be more pivotal and discriminant within high-risk groups, as characteristics such as age may be less predictive of vaccination in samples of over 65 s and health status may be less important in samples of younger people with eligible health conditions.

Our results also show that practical barriers were not important, except for time in the USA. This finding suggests that a culture of long working hours and short holidays may indeed have a negative effect on vaccination uptake.

Consistent with previous research, we found that physicians' opinion (and relatives' opinion in the UK), perceived vulnerability to and likelihood of influenza (and severity of influenza measured in number of bed-days in the UK), perceived vaccine effectiveness (only in the USA), the perception that the vaccine transmits influenza (in the USA and UK) or that its contents are dangerous (France), and perceived vaccine-related self-efficacy (UK) were associated with vaccine uptake. ${ }^{9-1225}$ As previously reported in the literature, ${ }^{11}$ we also found a small negative association between the perceived severity of influenza and past vaccination in the USA, and no association in the UK and France. A possible explanation is that people who believe that influenza could make them severely ill, may also be concerned about the vaccine influenza-like symptoms, thus omission bias may induce them to refrain from vaccinating. ${ }^{31} 47$ Alternatively, the knowledge that influenza could be serious may not necessarily translate into a feeling of personal threat, particularly among younger individuals. A similar result was the lack of or negative association between perceived susceptibility to influenza and past vaccination in the USA and France, and the UK, respectively. These findings indicate that measuring perceived influenza severity as degree of seriousness ('the flu could make me 
Table 5 Survey items associated with past influenza vaccination

\begin{tabular}{|c|c|c|c|}
\hline Item & USA & UK & France \\
\hline What is your date of birth? & & & $\checkmark$ \\
\hline Have you ever been diagnosed with any of the following (eligible) conditions? & $\checkmark$ & $\checkmark$ & \\
\hline Do you have a private health insurance? & $\checkmark$ & $\checkmark$ & $\checkmark$ \\
\hline Do you have public health insurance (eg, Medicare)? & $\checkmark$ & & \\
\hline What is your gender? & & $\checkmark$ & \\
\hline Which of the following options best describe your current situation (marital status)? & & $\checkmark$ & $\checkmark$ \\
\hline What is your combined annual household income? & $\checkmark$ & & \\
\hline What is the highest level of education you have completed? & & & $\checkmark$ \\
\hline I can make time to get the flu vaccine. & $\checkmark$ & & \\
\hline My physician thinks I should get a flu vaccine. & $\checkmark$ & $\checkmark$ & $\checkmark$ \\
\hline My relatives or close friends think I should get a flu vaccine. & & $\checkmark$ & \\
\hline With no flu vaccine, I would feel very vulnerable to the flu. & $\checkmark$ & $\checkmark$ & \\
\hline If I got the flu, I would feel sicker than other people my age. & & $\checkmark$ & \\
\hline Without a flu vaccine, I am sure I would get the flu this winter. & $\checkmark$ & $\checkmark$ & $\checkmark$ \\
\hline I believe that if I got the flu I would have to stay in bed for... & & $\checkmark$ & \\
\hline The flu could make me severely ill. & $\checkmark$ & & \\
\hline
\end{tabular}

The flu could make me severely ill.

If I don't get the flu vaccine and I get the flu, passing the flu to other people would worry me because it would be my fault.

I am confident I can avoid getting the flu, even without the flu vaccine.

If I don't get a flu vaccine and end up getting the flu this winter, I would regret not getting the vaccine.

I feel I know enough about the flu vaccine to make an informed decision about whether to get vaccinated or not.

If I get a flu vaccine, I will be protected against the flu.

The flu vaccine could give me the flu.

I am worried that some of the contents of the flu vaccine may be dangerous for me.

I am confident I can get a flu vaccine if I want one.

Which of the following statements best represent how much you trust your physician? (Trust scale)

How actively do you participate with your physician in making decisions about health, generally?

(Health decision-making scale)

I had a bad experience with vaccines or injections.

I had a scary health-related experience.

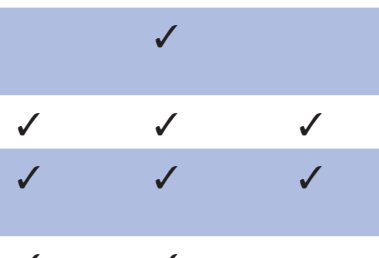

$\checkmark d$

$\checkmark d$

See the full list of included items and response categories in online supplementary table S1.

severely ill') and perceived susceptibility to influenza as individuals' constitutional vulnerability in relation to that of others ('If I got the flu, I would feel sicker than other people my age'), does not improve our understanding of vaccination behaviour, as previously suggested. ${ }^{11}$

Interestingly, perceived vaccine knowledge (to make informed decisions) was negatively correlated with past vaccination in the USA and positively correlated in the UK. Researchers have long advocated for strategies to increase knowledge about vaccines, ${ }^{10}$ yet these results suggest that a cognitive approach may not always be effective, particularly when the target population (eg,
USA unvaccinated people) perceive themselves as being knowledgeable, and hence are less likely to seek or be receptive to further information.

Factors which are less explored in the literature were also robustly correlated with past vaccination. Perceived control over influenza and regret of catching it (if unvaccinated) were significantly associated with past vaccination behaviour across the three countries. Worry of infecting other people (if unvaccinated) was only linked to past vaccination in the $\mathrm{UK}$, but the direction of the association was unexpected: unvaccinated participants worried more than vaccinated participants of infecting other people if 
they were to remain unvaccinated. Although this question was hypothetical, it is plausible that unvaccinated participants felt worried about infecting others because of their actual vaccination status, whereas vaccinated participants did not, either because they felt protected by the vaccine or they do not generally worry about infecting others. In any case, this result does not support the notion that altruism motivates people to vaccinate. ${ }^{27}$

Our results also show that trust in key vaccination stakeholders does not play a significant role in influenza vaccination decisions in these countries. In fact, we found that US vaccinees were less trusting of their physician than those who did not vaccinate. This finding conflicts with the premise that all vaccination decisions are a combination of individuals' perceptions of the information they receive and their trust in those who manufacture, legislate and deliver vaccines. ${ }^{26}$

A striking finding from a qualitative study ${ }^{31}$ held true when tested quantitatively. UK participants who had a bad experience with needles in childhood were less likely to vaccinate later in life, consistent with evidence showing that traumatic experiences can linger through to adulthood and significantly influence health decisions. ${ }^{48}$ This was further supported by the increased likelihood of vaccinating exhibited by those who reported a scary health-related experience in childhood across the three countries, although less so in the USA, possibly due to a lasting perception of vulnerability that resulted in enhanced preventive behaviours in adulthood. To our knowledge, this is the first quantitative study linking adult vaccination behaviour with childhood experiences. Therefore, further testing these results across different samples would be desirable to ensure that the link (or lack thereof) between these variables and influenza vaccination is a true one. Additionally, future research could unpack this synergistic effect using qualitative approaches.

Finally, we found that UK vaccinees were more likely to let their doctors make decisions about their health. This finding resonates with findings from Opel and colleagues which showed that parents were more likely to resist advice if the doctor used a participatory (eg, 'What do you want to do about shots?") rather than a presumptive initiation approach (eg, 'Well, we have to do some shots'). ${ }^{49}$ Researchers could test the replicability of Opel's study on adult vaccination and further explore the role of health decision-making preferences on doctor-patient communication about vaccines.

\section{Policy implications}

This study offers evidence that can inform policy and practice. Sociopsychological factors associated with influenza vaccination can be used to track vaccination sentiment and forecast uptake. These factors are currently not consistently monitored and rarely used as a basis for effective service delivery and communication strategies. If we are to improve or at least sustain current immunisation rates, we must start actively listening to the public by including these aspects into national immunisation surveys. An important challenge for policymakers is prioritising what to monitor and to what extent. As a first step, influenza vaccination surveillance systems should include the explanatory variables reported here, particularly those accounting for a significant proportion of the variance in vaccination behaviour (ie, social influence and influenza perceptions), and make additions or adjustments over time.

More importantly, our findings suggest that sociopsychological factors could provide a valuable opportunity to develop and evaluate targeted interventions to improve vaccination coverage. For instance, the influence of physicians' opinions on vaccination, over and above people's trust in immunisation stakeholders (including physicians themselves), indicates that improving communications at the practice level should be prioritised. One possible intervention is to reach undervaccinated groups (eg, younger eligible individuals) via consultations and vaccination reminders, a strategy that has been successful in older populations..$^{50} \mathrm{~A}$ complementary initiative is to link influenza vaccination rates to pay-for-performance systems, such as the UK Quality and Outcomes Framework which rewards GPs for vaccinating some at-risk groups. Yet, further incentivising primary care practices to employ more effective approaches to reach out to eligible unvaccinated patients may require a stratified strategy that offers larger rewards for vaccinating subgroups with low vaccination rates and additional incentives for exceeding vaccination targets. ${ }^{51}$ However, we acknowledge that the implementation of more complex incentive systems would require additional resources. In the USA, programmes to introduce the influenza vaccine in the workplace may encourage those with limited time to protect themselves.

Efforts could also focus on addressing the gap between perceived and real risks of influenza. This could be achieved by moving away from generic messages about the threat of influenza (eg, 'influenza is serious') towards tailored messages which take into consideration the needs and characteristics of different at-risk populations. For instance, influenza-related complications in young diabetics may differ from those experienced by elderly people. Specific messages may, therefore, allow individuals and their families to better identify risks relevant to their condition and, in turn, compel them to vaccinate.

Similarly, effective communications as part of the consultation aimed at assuaging concerns around vaccines could take into account decision-making preferences and individual past experiences, particularly in the UK. For instance, communication efforts are likely to be better spent on those who prefer to make decisions about their heath independently than those who are more prone to delegate health decisions to their physician. Given the lasting effect of some traumatic childhood experiences, interventions and new products aimed at making all childhood encounters with injections as easy as possible may be a good investment in the success of vaccination programmes in the future. 
However, in a context of constrained resources, physicians and nursing staff have limited time and resources to improve vaccination services and communications. Hence, increased investment in the provision of training, adequate communication materials and decision aids to enhance patient-doctor communication is urgently needed and much deserved.

Messages delivered in primary care settings could also be complemented with evidence-based mass communications. For example, a national campaign could combine messages about the risks of influenza (eg, likelihood of catching it and feelings of vulnerability and regret for not vaccinating) with messages about the limited protectiveness of avoidance strategies (eg, taking vitamins or evading crowds), and provide-rather than avoid —easy-to-understand and accurate information about vaccine safety (eg, communicating more effectively the difference between vaccine-induced symptoms and actual influenza symptoms) and effectiveness, particularly in the USA. When possible, mass communications should also be tailored to specific at-risk populations.

Finally, given that the influenza vaccine is more effective in healthy working adults ${ }^{52}$-reducing the number of influenza-like episodes among this population, but also providing indirect protection to at-risk groups, knowing what motivates them to vaccinate can be valuable to policymakers seeking to reduce the societal cost of influenza.

\section{Limitations}

This study has several limitations, some of which may affect the generalisability of our findings. Although the use of non-probability online panels has become increasingly common, ${ }^{53}{ }^{54}$ response rates are generally low. ${ }^{3755}$ This is largely because online panel members become desensitised to survey email invitations from the online panel provider. ${ }^{556}$ However, an emerging body of evidence shows that higher response rates may not be associated with more accuracy, in fact, some studies have found that high response rates can yield less accurate results. ${ }^{57}$ This suggests that the low response rates we achieved may not be as important a source of bias as using a sample drawn from a non-probability online panel. This is because the relationship between the sample and the non-probability online panel population is often unknown, so it is not possible to estimate how representative the sample is of the population as a whole. Therefore, our research may have suffered from respondent-related biases; for example, people for whom vaccination issues are particularly salient may have been more prone to participate.$^{56}$ Consequently, responses may have been more polarised, both in favour and against of vaccination. Future studies testing our findings using different sampling strategies, such as the use of probability online panels or random digit dialling, are warranted. A related limitation is that our US sample was more educated than the population, which may have affected the generalisability of our findings, although there is no consensus regarding the link between education and influenza vaccination in the USA. ${ }^{9} 10^{12}$ Similarly, in France, participants were less likely to disclose their household income and over half reported it to be equal or below the band that was closest to the median income of the population, which could also have biased our results. Further, since we sought to attain samples that were representative of the adult population, they may not have been adequately powered to detect subgroup differences (eg, whites vs non-whites).

Another possible drawback is that lengthy instruments may fatigue participants and affect the quality of the data. Although pilot results indicated that participants did not feel the survey was long or difficult to complete, there is a chance that those who did not finish the survey may have found it too lengthy. A related limitation is the dichotomisation of four continuous variables, which could have resulted in loss of information. However, on balance, this was deemed necessary to aid the analysis of survey items with numerous 'I don't know/not applicable' responses, which are not the same as missing responses. Strategies used to deal with missing responses, such as imputation or case exclusion, would have been inappropriate or would have significantly reduced the size of our samples and affected their composition.

An additional limitation is the use of a subjective outcome measure. Although data from medical records may be preferable, previous research comparing the accuracy of the latter to self-reported influenza vaccination has shown these can coincide in up to $90 \%$ of the cases ${ }^{58}$ Further, since some people vaccinate at work or alternative facilities such as pharmacies, it remains unclear whether medical records are more accurate than self-reports.

Lastly, although we employed a battery of measures designed to capture people's actual perceptions about influenza and the influenza vaccine, using a prospective design and a representative sample of vaccine-naïve participants would have been preferable to avoid postdecisional rationalisations. However, this research design requires substantial financial resources and time which were not available to us, and hence, a retrospective design was chosen instead. Consequently, and consistent with other retrospective cross-sectional studies, causation cannot be inferred, thus some of the assessed perceptions may have been generated or reinforced by prior vaccination. Moreover, this study's design precludes any attempt to predict future behaviours. Further research testing whether the identified explanatory variables prospectively predict actual vaccination uptake among first-time vaccinees is merited.

\section{CONCLUSIONS}

This study identifies policy amenable factors associated with past influenza vaccination and presents a set of robust explanatory variables that aims to attain a comprehensive and more accurate understanding of the constellation of 
factors underpinning vaccination behaviour. Our findings can prove useful for countries looking to improve vaccination rates by developing more opportune and effective communication strategies and implementing evidencebased interventions. Our results highlight the importance of routinely monitoring vaccination sentiment and using these data to inform immunisation policy.

Acknowledgements The authors would like to thank the funders of this study and all those who agreed to take part in our research. We are also grateful to Bruno Rigole (Sanofi Pasteur), Rebecca da Cunha, Brian Larkin, Sofia Lombera and Natasha Phillips (Double Helix), and Nicole Huyghe and Kerry 0'Neill (solutions-2) for their valuable advice and management support throughout this study.

Contributors All authors contributed to the design of the study, the interpretation of the results and write-up of the manuscript. AW and MM performed the analyses.

Funding This work was supported by Sanofi Pasteur through a research grant (P40006_WSSS) and the National Institute for Health Research Imperial Patient Safety Translational Research Centre (PSTRC) (RDPSC 79560), UK.

Competing interests This research is funded by the National Institute for Health Research (NIHR) via the Collaboration for Leadership in Applied Health Research and Care South London at King's College Hospital NHS Foundation Trust, London, UK. NS also delivers behavioural and safety interventions and training to hospitals internationally on a consultancy basis through London Safety and Training Solutions Ltd. AW and NS currently have a consultancy agreement as paid scientific advisors in the design of a shingles vaccine survey by Sanofi MSD. AT is employed by Sanofi Pasteur. MM and CV have nothing to disclose. The views expressed are those of the authors and not necessarily those of the National Health Service, the NIHR, the Department of Health or Sanofi Pasteur.

Patient consent Participants were members of the general public (not patients). As the survey was completed online, a standard consent form was filled in by participants electronically.

Ethics approval The American Institutes for Research (USA) and the Imperial College Research Ethics Committee (UK) granted research ethics approval. The French Commission nationale de l'informatique et deslibertés and Comités de protection des personnes granted waivers to approval.

Provenance and peer review Not commissioned; externally peer reviewed.

Data sharing statement There are no additional data available.

Open Access This is an Open Access article distributed in accordance with the Creative Commons Attribution Non Commercial (CC BY-NC 4.0) license, which permits others to distribute, remix, adapt, build upon this work non-commercially, and license their derivative works on different terms, provided the original work is properly cited and the use is non-commercial. See: http://creativecommons.org/ licenses/by-nc/4.0/

(c) Article author(s) (or their employer(s) unless otherwise stated in the text of the article) 2017. All rights reserved. No commercial use is permitted unless otherwise expressly granted.

\section{REFERENCES}

1. GBD 2013 Mortality and Causes of Death Collaborators. Global, regional, and national age-sex specific all-cause and causespecific mortality for 240 causes of death, 1990-2013: a systematic analysis for the Global Burden of Disease Study 2013. Lancet 2015;385:117-71.

2. Centers for Disease Control and Prevention (CDC). Estimates of deaths associated with seasonal influenza --- United States, 19762007. MMWR Morb Mortal Wkly Rep 2010;59:1057.

3. ECDC. Factsheet for the general public. http://ecdc.europa.eu/ en/healthtopics/seasonal_influenza/basic_facts/Pages/factsheet_ general_public.aspx (accessed 30 Mar 2015).

4. CDC. Seasonal influenza vaccination trends. http://www.cdc.gov/flu/ fluvaxview/trends.htm (accessed 1 Feb 2016).

5. PHE. Influenza immunisation programme for England: gp patient groups data collection survey season 2013 to 2014. https://www. gov.uk/government/uploads/system/uploads/attachment_data/file/ 319694/2902494_FluVaccineUptake_GPPatients2013-14_acc.pdf (accessed 1 Dec 2014).
6. 56th World Health Assembly Prevention and Control of Influenza Pandemics and annual epidemics. Geneva, Switzerland 2003.

7. ONS. Excess Winter Mortality in England and Wales, 2014/15 (Provisional) and 2013/14 (Final). http://webarchive.nationalarchives. gov.uk/20160105160709/http://www.ons.gov.uk/ons/rel/ subnational-health2/excess-winter-mortality-in-england-and-wales/ 2014-15-provisional-and-2013-14-final-/index.html (accessed 30 May 2016).

8. PHE. Influenza immunisation programme for England: gp patient groups data collection survey season 2015 to. $2016 \mathrm{https}$ ://www. gov.uk/government/uploads/system/uploads/attachment_data/file/ 526033/Seasonal_flu_GP_patient_groups_annual_report_2015_2016. pdf (accessed 30 May 2016).

9. Chapman GB, Coups EJ. Predictors of influenza vaccine acceptance among healthy adults. Prev Med 1999;29:249-62.

10. Kohlhammer Y, Schnoor M, Schwartz M, et al. Determinants of influenza and pneumococcal vaccination in elderly people: a systematic review. Public Health 2007;121:742-51.

11. Brewer NT, Chapman GB, Gibbons FX, et al. Meta-analysis of the relationship between risk perception and health behavior: the example of vaccination. Health Psychol 2007;26:136-45.

12. Wheelock A, Thomson A, Sevdalis N. Social and psychological factors underlying adult vaccination behavior: lessons from seasonal influenza vaccination in the US and the UK. Expert Rev Vaccines 2013;12:893-901.

13. Centers for Disease Control and Prevention (CDC). Influenza vaccination and self-reported reasons for not receiving influenza vaccination among Medicare beneficiaries aged $>$ or $=65$ years-United States, 1991-2002. MMWR Morb Mortal Wkly Rep 2004:53:1012-5.

14. Tversky A, Kahneman D. Availability: a heuristic for judging frequency and probability. Cogn Psychol 1973;5:207-32.

15. Weinstein ND. Misleading tests of health behavior theories. Ann Behav Med 2007;33:1-

16. NVAC. NVAC Vaccine Hesitancy Working Group Charge. http://www. hhs.gov/nvpo/nvac/subgroups/nvac-vaccine-hesitancy-wgcharge. html (accessed 28 Feb 2014).

17. Larson HJ, Jarrett C, Schulz WS, et al. SAGE Working Group on Vaccine Hesitancy. Measuring vaccine hesitancy: The development of a survey tool. Vaccine $2015 ; 33: 4165-75$.

18. Weinstein ND, Kwitel A, McCaul KD, et al. Risk perceptions: assessment and relationship to influenza vaccination. Health Psychol 2007;26:146-51.

19. Liao Q, Wong WS, Fielding R. Comparison of different risk perception measures in predicting seasonal influenza vaccination among healthy Chinese adults in Hong Kong: a prospective longitudinal study. PLOS One 2013;8:e68019.

20. Nexøe J, Kragstrup J, Søgaard J. Decision on influenza vaccination among the elderly. A questionnaire study based on the Health Belief Model and the Multidimensional Locus of Control Theory. Scand J Prim Health Care 1999;17:105-10.

21. Callegaro M, Baker RP, Bethlehem J, et al. Online Panel Research: a Data Quality Perspective. John Wiley \& Sons 2014.

22. Toepoel V, Das M, Soest V. Effects of design in web surveys: comparing trained and fresh respondents. Public Opin Quart 2008:72-985.

23. Rosenstock IM. Why people use health services. Milbank Mem Fund Q 1966;44:94-127.

24. Rogers RW. A Protection Motivation Theory of Fear Appeals and Attitude Change1. J Psychol 1975;91:93-114.

25. Liao Q, Cowling BJ, Lam WW, et al. Factors affecting intention to receive and self-reported receipt of 2009 pandemic $(\mathrm{H} 1 \mathrm{~N} 1)$ vaccine in Hong Kong: a longitudinal study. PLoS One 2011;6:e17713.

26. Larson HJ, Cooper LZ, Eskola J, et al. Addressing the vaccine confidence gap. Lancet 2011;378:526-35.

27. Shim E, Chapman GB, Townsend JP, et al. The influence of altruism on influenza vaccination decisions. J $R$ Soc Interface 2012;9:2234-43.

28. Wallston KA, Wallston BS, Smith $\mathrm{S}$, et al. Perceived control and health. Curr Psychol 1987;6:5-25.

29. Lehmann BA, Ruiter RA, Kok G, et al. A qualitative study of the coverage of influenza vaccination on Dutch news sites and social media websites. BMC Public Health 2013;13:742-51.

30. Chapman GB, Coups EJ. Emotions and preventive health behavior: worry, regret, and influenza vaccination. Health Psychol 2006;25:82-90.

31. Wheelock A, Parand A, Rigole B, et al. Socio-psychological factors driving adult vaccination: a qualitative study. PLoS One 2014;9:e113503.

32. Robinson A, Thomson R. Variability in patient preferences for participating in medical decision making: implication 
for the use of decision support tools. Qual Health Care 2001;10(suppl 1):i34-i38.

33. Safran DG, Kosinski M, Tarlov AR, et al. The Primary Care Assessment survey: tests of data quality and measurement performance. Med Care 1998;36:728-39.

34. Caille-Brillet A, Raude J, Lapidus N, et al. Trends in influenza vaccination behaviours-results from the CoPanFlu cohort, France, 2006 to 2011. High Educ 2013;419::26.6-31.

35. Saris WE, Gallhofer IN. Estimation of the effects of measurement characteristics on the quality of survey questions. In: Saris WE, Gallhofer IN, eds. Design, evaluation, and analysis of questionnaires for Survey Research. Hoboken, NJ: John Wiley \& Sons, 2007.

36. Hosmer Jr DW, Lemeshow S, regression Alogistic. John Wiley \& Sons, 2004.

37. Baker R, Blumberg SJ, Brick JM, et al. Research synthesis AAPOR report on online panels. Public Opin Quart 2010;74:711-81.

38. Sheldon $\mathrm{H}$, Graham C, Pothecary N, et al; Increasing response rates amongst black and minority ethnic and seldom heard groups. Picker Institute Europe, 2007.

39. Riphagen-Dalhuisen J, Gefenaite G, Hak E. Predictors of seasonal influenza vaccination among healthcare workers in hospitals: a descriptive meta-analysis. Occup Environ Med 2012;69:230-5.

40. ECDC. Seasonal influenza vaccination and antiviral use in Europe Overview of vaccination recommendations and coverage rates in the EU Member States for the 2013-14 and 2014-15 influenza seasons. Published 2016. (accessed 22 Aug 2016).

41. Friedman L, Wall M. Graphical views of suppression and multicollinearity in multiple linear regression. Am Stat 2005;59:127-36.

42. MacKinnon DP, Krull JL, Lockwood CM. Equivalence of the mediation, confounding and suppression effect. Prev Sci 2000;1:173-81.

43. Bowerman BL, O'Connell RT. Linear statistical models: an applied approach. Belmont, CA: Duxbury, 1990.

44. Field A. Discovering statistics using IBM SPSS statistics. 4th ed. London: Sage, 2013.

45. Cook RD, Weisberg S. Residuals and influence in regression. New York: Chapman \& Hall, 1982.

46. Peretti-Watel P, Raude J, Sagaon-Teyssier L, et al. Attitudes toward vaccination and the $\mathrm{H} 1 \mathrm{~N} 1$ vaccine: poor people's unfounded fears or legitimate concerns of the elite? Soc Sci Med 2014:109:10-18.

47. Ritov I, Baron J. Reluctance to vaccinate: omission Bias and ambiguity. J Behav Decis Mak 1990;3:263-77.

48. Taddio A, Katz J, llersich AL, et al. Effect of neonatal circumcision on pain response during subsequent routine vaccination. Lancet 1997;349:599-603.
49. Opel DJ, Heritage J, Taylor JA, et al. The architecture of providerparent vaccine discussions at health supervision visits. Pediatrics 2013;132:1037-46.

50. Thomas RE, Russell M, Lorenzetti D. Interventions to increase influenza vaccination rates of those 60 years and older in the community. Cochrane Database Syst Rev 2010;8:CD005188.

51. Dexter LJ, Teare MD, Dexter M, et al. Strategies to increase influenza vaccination rates: outcomes of a nationwide cross-sectional survey of UK general practice. BMJ Open 2012;2:e000851.

52. Legrand J, Vergu E, Flahault A. Real-time monitoring of the influenza vaccine field effectiveness. Vaccine 2006;24:6605-11.

53. Couper M. Web surveys: a review of issues and approaches. Public Opin Q 2000;64:464-94.

54. Pedersen MJ, Nielsen CV. Improving survey response rates in Online Panels: effects of Low-Cost Incentives and Cost-Free text Appeal Interventions. Soc Sci Comput Rev 2016;34:229-43.

55. Tourangeau R, Groves RM, Kennedy C, et al. The presentation of a web survey, nonresponse and measurement error among members of web panel. J Off Stat 2009;25:299-321.

56. Keusch $\mathrm{F}$. The role of topic interest and topic salience in online panel web surveys. International Journal of Market Research 2013;55:59-80.

57. Yeager DS, Krosnick JA, Chang L, et al. Comparing the Accuracy of RDD Telephone surveys and internet surveys conducted with probability and Non-Probability samples. Public Opin Q 2011;75:709-47.

58. Nichol KL, Korn JE, Baum P. Estimation of outpatient risk characteristics and influenza vaccination status: validation of a selfadministered questionnaire. Am J Prev Med 1991;7:199-203.

59. US Census Bureau. Statistical Abstract of the United States: 2012. 131st ed. Washington: DC, 20112011. http://www.census.gov/ compendia/statab/.

60. Office for National Statistics. Annual Mid-year Population Estimates 2013 http://www.ons.gov.uk/ons/dcp171778_367167.pdf (Accessed December 1, 2014).

61. Office for National Statistics. 2011 Census: aggregate data (England and Wales) [computer file: UK Data Service Census Support. http:// infuse.mimas.ac.uk. (accessed $1 \mathrm{Dec} 2014$ ).

62. Department for Work and Pensions Households Below Average Income - An analysis of the income distribution 1994/95 - 2012/13. https://www.gov.uk/government/uploads/system/uploads/ attachment_data/file/325416/households-below-average-income1994-1995-2012-2013.pdf (accessed 20 Apr 2015).

63. National Institute of Statistics and Economic Studies. Population census 2011. Published 2012. http://www.insee.fr/. (accessed 15 Apr 2015). 